\title{
Expression of uncertainty in fuzzy scales based measurements
}

\author{
Eric Benoit \\ LISTIC, Universite de Savoie, Annecy, France
}

\begin{abstract}
Fuzzy scales were introduced as a transition between weak scales and strong scales. Preceding studies on fuzzy scales considered only ideal exact measurement without any consideration of uncertainty. The goal of this paper is to present a general approach for the management of uncertainty within the context of fuzzy scale based measurements. After a short reminder on fuzzy scales, a method to define a probability density function or a possibility function on indications given by a fuzzy scale based measurement is exposed. Finally, a method based on the evidence theory is applied to build simultaneously a probability density function and an associated possibility function.
\end{abstract}

Keywords uncertainty, fuzzy scale, measurement science.

\section{Introduction}

The concept of fuzzy scale was introduced in order to add properties to nominal scales and ordinal scales [1]. By the way, it establishes a link between strongly defined measurements used for example in physical sciences and weakly defined measurements used in behavioural sciences as for example in psychophysics [2]. Actually, except in Physics, the Measurement Science is more and more concerned by weakly defined measurements. As examples, Psychophysics or Economy and more globally the measurement of complex systems are concerned.

Since their first introduction, fuzzy scales were exclusively used to perform a precise representation of a unique property manifestation. In a measurement context, it is now admitted that the representation of a property manifestation needs to expose the imprecision and the uncertainty related to the definition of the property and to the measurement process. Within the current metrological approach, a measurement result is given by a probability density function (PDF) that expresses a knowledge about the measurand. More recent approaches extend such knowledge representation with a possibility function (PF). Considering that the source of this knowledge is a set of indications, the computation of the PDF or of the PF is performed on the representation space including these indications. In usual measurements, this space is a numerical one. Within the context of fuzzy scales, a second stage 
in the measurement process performs a translation from a numerical representation of indications to a fuzzy lexical representation. The computation of PDF or PF may not depend on the representation space. By the way, it may be possible to define a function on a space and to translate it into another space. The issue in which this paper is concerned is that such functions are actually approximated with parametric functions. The accuracy of the approximation depends on the capability for a family of parametric functions to fit an original PDF or PF.

Our hypothesis is to consider that, even if quantities are part of the concrete world, they are defined within a model, itself respecting a theory. Within the context of weakly defined measurement, we consider that the definitional uncertainty of a quantity has to be expressed on the representational space. Then, when a measurement is performed with a fuzzy scale, the definitional uncertainty has to be defined on the associated representation space, i.e. on a space of lexical fuzzy subsets.

\section{Fuzzy scales}

Fuzzy scale are characterized by their capability to represent a similarity relation between manifestations with a fuzzy equivalence relation between quantity values. The quantity values are expressed as Lexical Fuzzy Subset (LFS), i.e. as fuzzy subsets of lexical terms. See for example in Fig. 1 the description of a manifestation with a Lexical Fuzzy Subset. They are recommended for weakly defined measurements on quantities that support similarity relations.

This new family presently includes the weakest fuzzy scales: the fuzzy nominal scales, and metrical fuzzy scales [3]. The last ones are fuzzy nominal scales enhanced with the definition of a distance. The fuzzy ordinal scales are still under studies. Expressing quantity values with LFSs can be performed by measurements with physical sensors, or with psychophysics experiments. For the first case, quantity values are first expressed on a conventional numerical space with a ratio scale or at least with an interval scale. At this step, empirical relations are mapped into numerical relations. Then a second translation maps the numerical expression of quantity values into a fuzzy lexical expression. The second mapping, based on a fuzzy scale, transfers only the fuzzy equivalence relation from the empirical relational set to the representational relational set in the case of a fuzzy nominal scale, and transfer a distance operator in case of a metrical scale. 


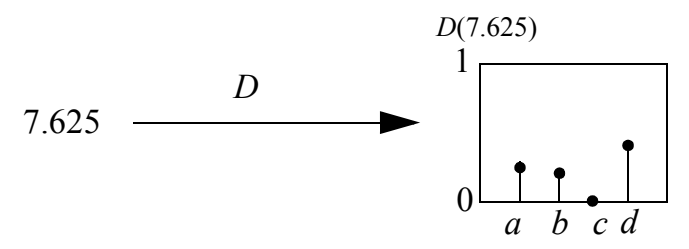

scalar lexical fuzzy subset

Fig. 1. Example of lexical fuzzy subset (LFS).

Fuzzy scales are defined by fuzzy symbolisation $<E, S_{T}, R,\{\sim\},\{=\},\{(\sim,=)\}>$ where:

- $E$ is the set of the manifestations of a quantity,

- $S_{T}$ is a set of terms used to qualify measurements,

- $R$ is a fuzzy relation on $E \mathrm{x} S_{T}$.

Let denote $F S\left(S_{T}\right)$ the set of fuzzy subsets of $S_{T}$, and $D$ the injective mapping defined as

$$
\begin{array}{cc} 
& D: E \rightarrow F S\left(S_{T}\right) \\
\forall e \in E \quad & D(e)=\left\{s \in S_{T} \mid e R s\right\}
\end{array}
$$

The relation $\sim$ is a fuzzy equivalence relation on $E$ that characterizes the scale. In this paper, $(\sim)(x, y)$ will denote the membership function of the relation $x \sim y$. This relation is homomorphic to a fuzzy equivalence relation $\sim$ on a subset $U \subset F S\left(S_{T}\right)$ such that $D(E) \subset U$ and $\sim$ is reflexive on $U:(\sim)(x, x)=1$. In previous studies, we proposed a familly of fuzzy equivalence relations denoted $(\Sigma-T)$ that holds the reflexivity property and a weak transitivity property [4]. The general form is given by Eq. (2) where $T$ is a t-norm.

$$
\forall(x, y) \in U^{2},(\sim)(x, y)=(\Sigma-T)(x, y)=\sum_{s \in S_{T}} T(x(s), y(s))
$$

The reflexivity condition induces a constraint on the set $U$

$$
\forall x \in U, \sum_{s \in S_{T}} T(x(s), x(s))=1
$$

The special cases with $T_{m}(a, b)=\min (a, b)$, see Eq. (4), and $T_{p}(a, b)=a . b$, see Eq. (5), were successfully applied to build fuzzy nominal scales.

$$
\begin{aligned}
& \forall(x, y) \in U^{2},(\sim)(x, y)=\left(\Sigma-T_{m}\right)(x, y)=\sum_{s \in S_{T}} \min (x(s), y(s)) \\
& \forall(x, y) \in U^{2},(\sim)(x, y)=\left(\Sigma-T_{p}\right)(x, y)=\sum_{s \in S_{T}} x(s) y(s)
\end{aligned}
$$


The constraint on $U$ given by the reflexivity property of $\sim$ become respectively:

$\forall x \in U, \sum_{s \in S_{T}} T_{m}(x(s), x(s))=\sum_{s \in S_{T}} x(s)=1$

and

$$
\forall x \in U, \sum_{s \in S_{T}} T_{p}(x(s), x(s))=\sum_{s \in S_{T}} x(s)^{2}=1
$$

From a mathematical point of view, the measurement values, expressed on $F S\left(S_{T}\right)$, are elements of a subset of a $\left|S_{T}\right|$ dimensional unit cube where $\left|S_{T}\right|$ is the cardinality of $S_{T}$.

\section{Modelling uncertainty}

\subsection{Model defined on a numerical space}

In a first trivial approach, the set of the numerical expression of the observations, i.e. the set of quantity values expressed in a numerical space, is used to produce a measurement result defined on this numerical set. The usual way is to define a probability density function (PDF) that models the set of quantity values (see GUM). An approximation of the probability density function by a parametric function allows analytical computation on PDFs. Another way also compatible with GUM is to model the set of quantity values with a possibility function (PF) that supports also the approximation with parametric functions [5]. The second stage is to perform a projection of this function on the set of LFSs. The result is a PDF (or a possibility function) on LFSs.

The advantage of this approach is the possibility to use the existing mathematical tools to create the PDFs or the possibility functions on a numerical space. A disadvantage is that the fuzzy scales need a lot of parameters to be defined [3]. Then a function defined on a numerical space with few parameters is mapped on the fuzzy lexical space into a function with a lots of parameters. In this case, the parametrization which role is to simplify the expression of a function, becomes useless. Another disadvantage is the impossibility to use this method for psychophysical measurements due to the fact that the numerical stage is avoided.

\subsection{Models defined on LFSs}

In a second approach, each observation is first expressed as a Lexical Fuzzy Subset of terms. Then a parametric function is created on LFSs to represent the set of quantity values. As mentioned in previous studies, the set of relations and operators that can be used to define the parametric functions is limited by the nature of the scale. For fuzzy nominal scales, the available relation is a fuzzy equivalence relation $\sim$. The metrical fuzzy scale defines a distance between LFSs. Let first define a general family of parametric functions on LFSs when the measurement 
is performed with a fuzzy nominal scale.

Definition 1: The function $f_{h, m}$ defined bellow is a probability density function on $U$ with mean $m$.

- Let $<E, S_{T}, R,\{\sim\},\{=\},\{(\sim,=)\}>$ be a fuzzy nominal scale.

- let $m \in U$ be a lexical fuzzy subset called mean,

- let $h$ be a monotonic decreasing function with domain $[0,1]$ and codomain $[0,+\infty[$.

- $f_{h, m}$ is a parametric function with domain $U$, codomain $[0,+\infty[$ and defined by

$$
f_{h, m}(x)=\frac{h(1-(\sim)(x, m))}{\sum_{. \sim-I T} h(1-(\sim)(y, m))}
$$

A PDF $f_{h, m}$ is defined with respect of the constraints of fuzzy nominal scales. The only relation involved into the definition is the fuzzy equivalence relation.

The concept of standard deviation is based on an aggregation of the differences with the mean. Into the set of LFSs, such difference is given by the equivalence relation: $(1-(\sim)(x, m))$. Then an expression of the standard deviation is given by

$$
\sigma=\sqrt{\sum_{x \in U}(1-(\sim)(x, m))^{2} f_{h, m}(x)}
$$

For example, the simplest PDF family is given by $f_{E_{a}, m}$ where $E_{a}$ is the step function defined by Eq. (8). The PDF $f_{E_{a}, m}$ defines a uniform distribution on lexical fuzzy subsets.

$$
E_{a}(b)=\left\{\begin{array}{lll}
1 & \text { if } & b \leq a \\
0 & \text { if } & b>a
\end{array}\right.
$$

Definition 2: The function $g_{h, m}$ defined bellow is a possibility distribution on $U$ which kernel includes $m$.

- Let $<E, S_{T}, R,\{\sim\},\{=\},\{(\sim,=)\}>$ be a fuzzy nominal scale,

- let $m \in U$ be a lexical fuzzy subset called mean,

- let $h$ be a monotonous decreasing function with domain $[0,1]$ and codomain $[0,+\infty[$.

- $g_{h, m}$ is a parametric function with domain $U$, codomain $[0,+\infty[$ and defined by

$$
g_{h, m}(x)=\frac{h(1-(\sim)(x, m))}{\max (\{h(1-(\sim)(y, m)) \mid(y \in U)\})}
$$


We can simply verify that $(1-(\sim)(x, y))$ is a distance with a saturation effect. The preceding definitions can be extended to the case of metrical fuzzy scales. A metrical fuzzy scale is defined by its symbolisation $<E, S_{T}, R$, $\{\sim, d\},\left\{=, d_{S}\right\},\left\{(\sim,=),\left(d, d_{S}\right)\right\}>$ such that $<E, S_{T}, R,\{\sim\},\{=\},\{(\sim,=)\}>$ is a fuzzy nominal scale, $d_{S}$ is a distance between terms $s$ of $S_{T}$, and $d$ is a distance on manifestations homomorphic to a distance on $U$.

Definition 3: The function $f_{h, m}$ defined bellow is a probability density function on $U$ with mean $m$.

- Let $<E, S_{T}, R,\{\sim, d\},\left\{=, d_{S}\right\},\left\{(\sim,=),\left(d, d_{S}\right)\right\}>$ be a metrical fuzzy scale

- Let $m \in U$ be a parameter.

- Let $h$ be a monotonous decreasing function with domain $[0,+\infty]$ and codomain $[0,+\infty[$.

- Let $d$ be the distance on $U$ that characterizes the metrical fuzzy scale.

- $f_{h, m}$ is a parametric function with domain $U$, codomain $[0,+\infty[$ and defined by

$$
f_{h, m}(x)=\frac{h(d(x, m))}{\sum_{\cdots-I_{T}} h(d(y, m))}
$$

Definition 4: The function $g_{h, m}$ defined bellow is a possibility distribution on $U$ which kernel includes $m$.

- Let $<E, S_{T}, R,\{\sim, d\},\left\{=, d_{S}\right\},\left\{(\sim,=),\left(d, d_{S}\right)\right\}>$ be a fuzzy nominal scale,

- let $m \in U$ be a lexical fuzzy subset called mean,

- let $h$ be a monotonous decreasing function with domain $[0,1]$ and codomain $[0,+\infty[$.

- $g_{h, m}$ is a parametric function with domain $U$, codomain $[0,+\infty[$ and defined by

$$
g_{h, m}(x)=\frac{h(d(x, m))}{\max (\{h(d(y, m)) \mid(y \in U)\})}
$$

At this step, we can conclude that with a distance, or at least a fuzzy equivalence between Lexical Fuzzy Subsets, it is possible to define parametric PDFs or PFs centred on a Lexical Fuzzy Subset.

The available methods to define the parameters are similar to the well known methods used on numerical spaces. Concerning the translations between PDFs and possibility functions, a general approach based on the DempterShafer evidence theory [6] is directly applicable.

\section{Construct method for PDF and PF}

\subsection{Evidence theory}

The Dempster-Shafer evidence theory, also named the transferable belief model (TBM) is a general approach for 
uncertainty management based on the assignment of probabilities to sets. It generalises both the probability theory and the possibility theory [7]. The main element of the TBM is the frame of discernment denoted $\Omega$ and presented as a set of elementary events, or as a discourse set for the expression of the belief. The knowledge is then expressed with a mapping denoted $m$ and called belief function from the set of subsets of $\Omega$, denoted $2^{\Omega}$, to the set $[0,1]$. This mapping represents the distribution of a weight of belief over the elements of $2^{\Omega}$. It verifies:

$$
\begin{gathered}
m(\varnothing)=0 \\
\sum_{A \in 2^{\Omega}} m(A)=1
\end{gathered}
$$

The set $\{A \mid m(A)>0\}$ is called the set of focal elements of $m$, and the couple $(\{A \mid m(A)>0\}, m)$ is called a body of evidence of a variable with values on $\Omega$. Fig. 2 gives the graphical semantic chosen in this paper to represent the body of evidences. For a given body of evidence, the associated set of focal elements is simply represented by a set, and the associated belief function is represented by a surface which projection is the set of focal elements. Two measures $\mathrm{Bel}$ and $P l$ are defined on the subsets of $\Omega$.

$$
\begin{aligned}
& \operatorname{Bel}(A)=\sum_{B \subseteq A} m(B) \\
& \operatorname{Pl}(A)=\sum_{B \cap A \neq \varnothing} m(B)
\end{aligned}
$$

When the focal elements are singletons, then the belief function is qualified as Bayesian. In this case, the two measures $\mathrm{Bel}$ and $\mathrm{Pl}$ are equal to a probabilistic measure $P$. The belief function $m$ is then equivalent to an assignment of probabilities to individual values, i.e. equivalent to a PDF.

When the focal elements are sets ordered by the inclusion operator, called nested sets or consonant sets, $\mathrm{Bel}$ and $\mathrm{Pl}$ are respectively the necessity measure $N$ and the possibility measure $\Pi$ of a possibility distribution derived from $m$.

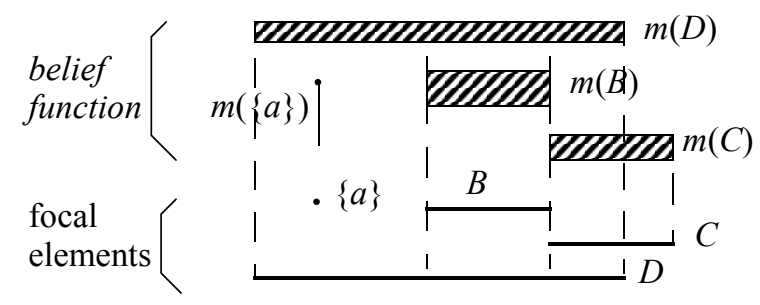

Fig. 2. Semantic used in this paper to represent belief functions. As a mass function, the belief function is represented by a surface

In [6] Yamada proposed a probability-possibility transformation based on the evidence theory. The principle is to identify a set of disjoint subsets of $\Omega$ computed from a PDF, then to compute a new body of evidence made of 
consonant subsets. We propose in this paper to build a parametric set of disjoint subsets in order to model a PDF within the framework of evidence theory. Then the Yamada's approach is applied to build the corresponding possibility distribution.

Within the context of metrology, the frame of discernment is the set all individual measurement values, and a measurement result is interpreted as a body of evidence of a quantity. It expresses the known representational information about a quantity.

\subsection{Construct method}

Let $S \subset U$ a finite set of measurement values, being the frame of discernment. Let $s_{0} \in S$ being a measurement value that will play the role of mean value.

Let $F=\left\{S_{i} \mid 1 \leq i \leq n\right\}$ being an ordered family of $n$ non-empty subsets of $S$ defined by $S_{i}=\left\{s \mid d_{i} \leq d\left(s, s_{0}\right)<d_{i+1}\right\}$.

Where $d$ is a distance on $U$ as defined in Chap. 3.2. and $d_{1}=0$.

An analogous definition on an Euclidean plane will define a family of rings, all centred on $s_{0}$. Fig. 3 and Fig. 4 give some examples of an ordered family of rings respectively in the case of a non-Euclidean metric space, and in the case of a mono-dimensional space.

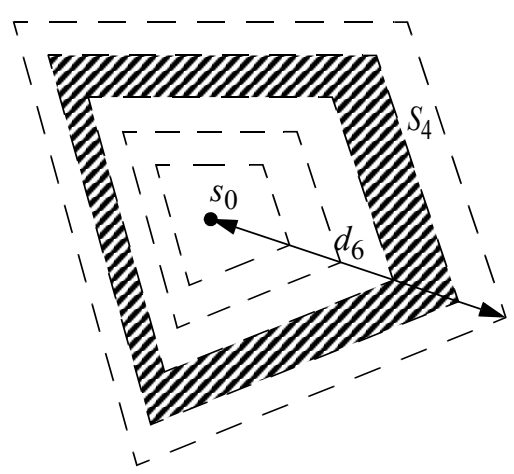

Fig. 3. Example of an ordered set family centred on $s_{0}$ and built with a non-Euclidean distance.

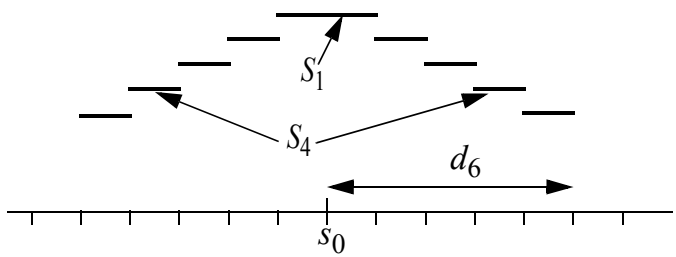

Fig. 4. Another example on a mono-dimensional space

Let $F^{P}$ a set of singletons defined by 
$F^{P}=\left\{\{s\} \mid s \in S_{1} \cup S_{2} \cup \ldots \cup S_{n}\right\}$

Let $m_{p}$ denotes a Bayesian belief function defined by: $m_{p}\left(\{s\} \mid s \in S_{i}\right)=h\left(d_{i}\right)$ where $h$ is a monotonous decreasing function with domain $[0,+\infty]$ and codomain $[0,+\infty[$ that verifies

$$
\sum_{i=1}^{n} h\left(d_{i}\right) \cdot\left|S_{i}\right|=1
$$

and $h\left(d_{n+1}\right)=0$,

where $\left|S_{i}\right|$ denotes the cardinality of $S_{i}$.

We can remark that $\left(F^{P}, m_{p}\right)$ is a body of evidence defining a probability distribution (see Fig. 5).

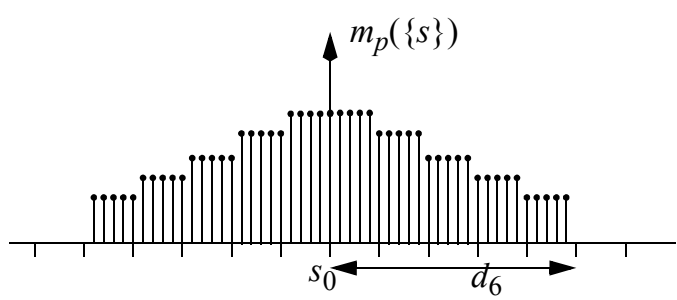

Fig. 5. Belief function $m_{p}$ defined on the singleton set $F^{P}$

Let now follow the Yamada's approach. A new family of sets is built from the family $F$.

$$
\begin{aligned}
& F^{\pi}=\left\{F_{k}^{\pi} \mid 1 \leq k \leq n\right\} \\
& \text { where } F_{k}^{\pi}=\bigcup_{h=1}^{k} S_{h}
\end{aligned}
$$

By definition these sets are nested. It is then possible to define a belief function $m_{\pi}$ which plausibility measure $P l$ is a possibility measure $\Pi$ (see Fig. 6).

$$
\begin{gathered}
m_{\pi}\left(F_{k}^{\prime \prime}\right)=\left|F_{k}^{\prime \prime}\right|\left(h\left(d_{k}\right)-h\left(d_{k+1}\right)\right) \\
=\left(\sum_{h=1}^{k}\left|S_{h}\right|\right)\left(h\left(d_{k}\right)-h\left(d_{k+1}\right)\right)
\end{gathered}
$$

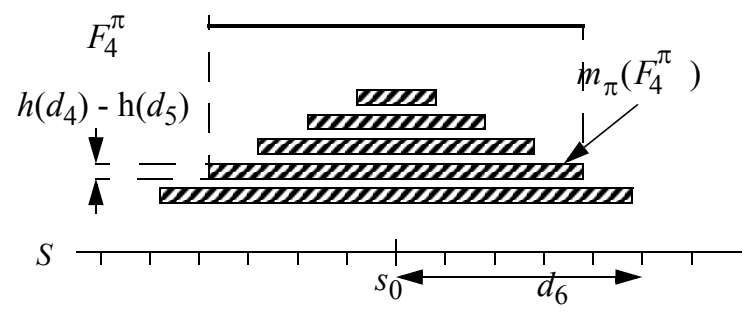

Fig. 6. Belief function $m_{\pi}$ defined on the set $F^{\pi}$ 
The possibility distribution is directly deduced from the belief function $m_{\pi}$.

$$
\begin{aligned}
& \pi(s)=\Pi(\{s\})=\operatorname{Pl}(\{s\})=\sum_{B \cap\{s\} \neq \varnothing} m_{\pi}(B) \\
& \forall s \in S_{k}, \pi(s)=\sum_{h=k}^{\cdot} m_{\pi}\left(F_{h}^{\pi}\right) \\
& =\left|F_{k}^{\pi}\right| h\left(d_{k}\right)+\sum_{i=k+1}^{n}\left|S_{j}\right| h\left(d_{j}\right)
\end{aligned}
$$

\subsection{Definition of the parameters}

With the chosen approach, all information about the probabilistic or the possibilistic representation of uncertainty on the space of LFSs is included into the ordered family $S_{i}=\left\{s \mid d_{i} \leq d\left(s, s_{0}\right)<d_{i+1}\right\}$ and on the mapping $h$. The procedure to define this information is

- to acquire a set of statistical data

- to translate this data into LFSs

- to extract the mean $s_{0}$ from the set of data

- to build a set family $F$

- to create an histogram from data mapped into $F$

- to compute the mapping $h$

The value $s_{0}$ has the same semantic than the mean in usual PDFs defined on interval scales or ratio scales. Due to the scales used in our case, the addition operator is not allowed for computations in the set of LFSs. So the mean cannot be computed with the usual formula. The value $s_{0}$ is then obtain by minimization of the cumulative distance to the data.

The set family $F$ is also characterized by a distance $d$ on LFSs and a set $\left\{d_{i} \mid 1 \leq i \leq n+1\right\}$. The distance $d$ is induced by the scale and cannot be considered as a parameter except for a calibration process.

The set $\left\{d_{i} \mid 1 \leq i \leq n+1\right\}$ is related to discrete aspect of the method. The influence of this information the PDF or the $\mathrm{PF}$ is small and, in a first approximation, it can be reduced to the parameters $\Delta d$ and $n$. The set become $\left\{d_{i}=(\mathrm{i}\right.$ $-1) \Delta d \mid 1 \leq i \leq n+1\}$.

Finally, $h$ is the main source of parametrization. It can be defined as a continuous parametric function, or as a discrete set of $n$ values. The usual statistical methods are then applied to estimate $h$ parameters. 


\section{Conclusion}

This paper shows that, with fuzzy nominal scale and metrical fuzzy scales, the definitional uncertainty can be managed in the case of weakly defined measurements. It provides a method to represent uncertainty on fuzzy lexical sets with the two main frameworks that are the probability theory and the possibility theory. The DempsterShafer theory used as a mainframe for these two theories, allows to compute simultaneously a possibility distribution and an associated a probability distribution.

The capability to manage uncertainty directly on fuzzy lexical sets is an important advance for behavioural sciences that cannot express their measurement result on numerical spaces.

\section{References}

[1] E. Benoit, L. Foulloy, Towards fuzzy nominal scales, Measurement 34(1) (2003) 49-55.

[2] L. Finkelstein, Widely, strongly and weakly defined measurement, Measurement 34(1) (2003) 39-48.

[3] E. Benoit, An ordinal metrical scale built on a fuzzy nominal scale, in: Physics Conference Series, Vol 238, 13th International Measurement Confederation TC1-TC7 Joint Symposium (IMEKO 2010), London, UK, id 012034, 7 pages, 2010.

[4] L. Foulloy, E. Benoit, Building a class of fuzzy equivalence relations, Fuzzy Sets and Systems, 157 (2006) $1417-1437$.

[5] D. Dubois, L. Foulloy, G. Mauris, H. Prade, Probability-Possibility Transformations Triangular Fuzzy Sets and Probabilistic Inequalities, Reliable Computing 10(4) (2004) 273-297.

[6] K. Yamada, Probability-possibility transformation based on evidence theory, Joint 9th IFSA World Congress and 20th NAFIPS International Conference, Vancouver, BC, Canada, 2001, Vol. 1, 70-75.

[7] P. Smets, Data fusion in the transferable belief model, in: Proc. of the 3rd Int. Conf. on Information Fusion (Fusion 2000), Paris, France, July 2000, Vol. 1, pp PS21-PS33.

Author: BENOIT Eric, LISTIC, Polytech Annecy-Chambery, University of Savoie, B.P. 80439, 74944 Annecy le Vieux Cedex, France, eric.benoit@univ-savoie.fr 